\title{
Editorial
}

\section{Surgery for Gastric Cancer: Has the East versus West Issue Been Solved?}

\author{
Yasuhiro Kodera \\ Department of Gastroenterological Surgery, Nagoya University Graduate School of Medicine, Nagoya, Japan
}

Authorities from the West and East contributed equally to complete this special issue of Digestive Surgery on gastric cancer. Given the incidence rates, the vast difference in cost and effort exerted to screen for this disease between the Far East and the rest of the world would seem inevitable. This explains the prominent difference in the proportion of early-stage disease among the regions, which apparently led to different opinions regarding some aspects of gastric cancer treatment. For instance, numerous mucosal cancers of the poorly differentiated phenotype located in the lower or middle portion of the stomach are found in the Far East. No surgeon would dream of considering total gastrectomy as a routine procedure for such a disease. Apparently, the concept of performing total gastrectomy routinely for poorly differentiated cancers as mentioned by de Steur et al. [this vol., pp. 96-103] arose in the West because cancers of this phenotype more often develop into large tumors and meticulous care is required to avoid cancer-positive resection margins. In addition, the terms and definitions used in the Japanese manuals such as the Japanese Classification of Gastric Carcinoma (JCGC) can sometimes be misleading when used by Western researchers. The Japanese manuals, convenient tools for those who wish to discuss gastric cancer treatment in detail, are compiled with considerable effort by the authorities and undergo frequent revision. Efforts have been exerted to distribute the synopsis of the revisions $[1,2]$, but these are not sufficient to

\section{KARGER}

E-Mail karger@karger.com

www.karger.com/dsu pass on the information to all of the world's experts in a timely fashion. Japanese surgeons are trained to purchase and use the new editions promptly, lest they will not be able to discuss gastric cancer using the common language at domestic conferences and meetings. It is important for the readers of this special issue to recognize these problems to further enhance their understanding of several excellent review articles written by the invited authors.

As an example, I would especially focus on the D numbers which denote the extent of lymphadenectomy. Some references to the D number by de Steur et al. [this vol., pp. 96-103] are outdated, but this should not be criticized. The JCGC is a manual that contains all the knowledge necessary to deal with the resected specimens to classify cancer according to the stage and histology, classify the curability of surgery and the extent of lymphadenectomy, and evaluate the response to the chemotherapy. This manual was first published in 1962 and has been revised regularly. The Japanese classification scheme for the extent of nodal dissection (D numbers) was revised substantially in 1999, after which lymphadenectomy was classified into three categories (D1, D2, and D3) instead of four (D1 to D4). Super-extended lymphadenectomy including systemic dissection of the para-aortic nodes (PAND) had been classified as D4 before 1999 (12th edition), but became D3 after 1999 (13th edition). This could be misleading when discussing the Taiwanese D1 versus D3 trial [3]. D3 in that trial is based on the 12th edition
(C) 2013 S. Karger AG, Basel

0253-4886/13/0302-0092\$38.00/0
Yasuhiro Kodera, MD, PhD, FACS

Department of Gastroenterological Surgery (Surgery II) Nagoya University Graduate School of Medicine 65 Tsurumai-cho, Showa-ku, Nagoya, Aichi 466-8550 (Japan) E-Mail ykodera@med.nagoya-u.ac.jp 
Fig. 1. Lymph node stations to be dissected when performing total gastrectomy (left) and distal gastrectomy (right). D1 requires dissection of the stations encircled in blue. Stations encircled in red need to be additionally resected to be defined as D2.
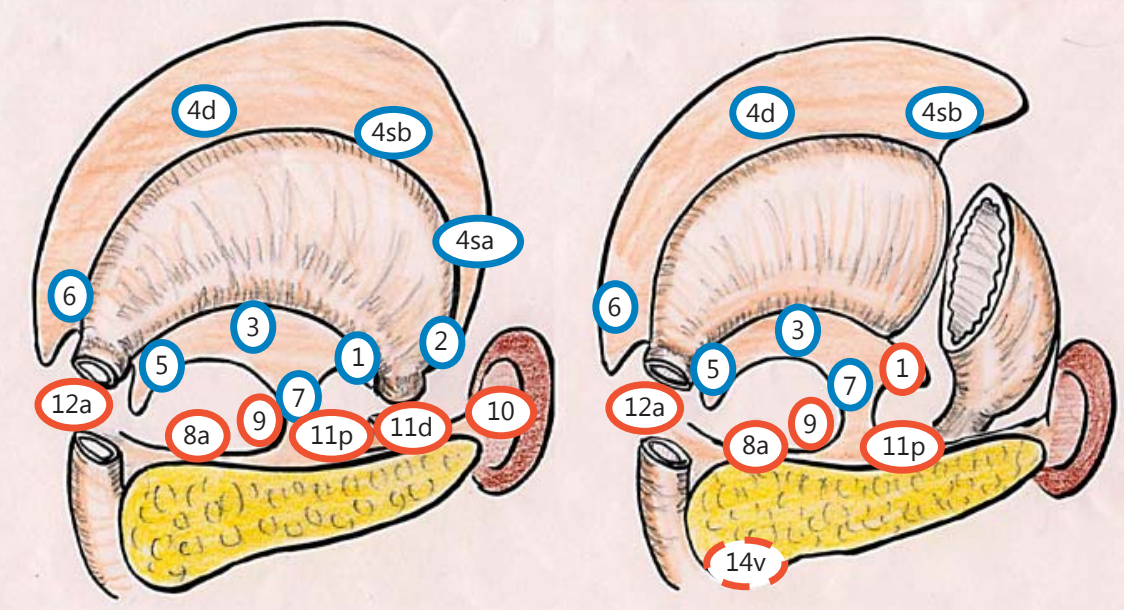

and more or less corresponds to D2 dissection in the modern era. Thus, it is more appropriate for most readers to consider the trial as a D1 versus D2 comparison, similar to the trials conducted in the Netherlands and Britain.

Up to the 13th edition of the JCGC, the D numbers corresponded to the anatomical site of the nodes to be dissected, which are expressed as $\mathrm{N}$ categories so that D1 dissection requires systemic dissection of $\mathrm{N} 1$ nodes. To make things simple, $\mathrm{N} 1$ nodes could be considered as the perigastric nodes as mentioned by de Steur et al. [this vol., pp. 96-103], but in reality, borderlines of the $\mathrm{N}$ categories shifted in accordance with the location of the tumor. For example, lymph nodes at station No. 2 (left paracardial nodes) should anatomically be considered as the perigastric nodes and were actually rated as N1 if the cancer was located in the upper third of the stomach, but they were regarded as distant nodes outside of the $\mathrm{N}$ categories for cancers located in the antrum. $\mathrm{N}$ categories at that time were complex even for the Japanese surgeons who were supposed to decide on which node stations to dissect based on knowledge of the $\mathrm{N}$ categories. Due to the complexity of the $\mathrm{N}$ categories and the corresponding D numbers, Siewert et al. [4] proposed a tentative classification in which the $\mathrm{D}$ numbers were based on the number of lymph nodes removed. D2 dissection in this context denotes radical gastrectomy with the removal of more than 25 lymph nodes. Although de Steur et al. [this vol., pp. 96-103] never referred to the D categories along that interpretation, it could be easy for surgeons in the West to wrongly do so.

To make things more complicated, the Japanese Treatment Guidelines for Gastric Cancer was compiled in 2002
[5] to provide an updated algorithm of the evidencebased treatments reflecting the new body of evidence obtained through phase III trials. In 2010, these two manuals underwent revision together for the first time, and the 14th edition of JCGC and the 3rd edition of the Guidelines were compiled. At this time, the authorities decided to intentionally split the JCGC and the Guidelines so that they would have different roles. The JCGC was to be used to describe the status of a tumor (histology, stage classification, etc.) and to evaluate treatment outcome (response, amount of residual disease, etc.), whereas the Guidelines were to define the treatment method (such as the D numbers) and to clarify the indication for various treatment options. D numbers are substantially simplified in the current version of the Guidelines so that the nodes to be removed depend on the extent of gastric resection, whether the patient undergoes total gastrectomy or distal gastrectomy (fig. 1). The new D classification is therefore much simpler and is expected to remain that way for some time so that doctors outside of Japan can share the concept without further confusion.

The concept of D2 dissection was perhaps first proposed by the Western surgeons who claimed that anatomically more thorough surgery could improve the outcome of gastric cancer [6]. However, the Japanese surgeons were the ones who successfully established and standardized the actual surgical procedure, thanks in no small measure to the lean patients more often found in Japan in the 1960s. Since then, D2 dissection has long been a standard there. The 'classic' D2 dissection has been tailored through extensive examination of the resected specimens and analysis of the long-term outcome. Japa- 
nese surgeons meticulously dissect specimens after surgery and harvest the lymph nodes from various lymph node stations, putting them into small bottles containing formalin - one bottle for each lymph node station. They do not dump the specimen into a bucket filled with formalin and leave the remaining procedures to the pathologists. Through these efforts to scrutinize the specimens along with routine application of D2 dissection, Japanese surgeons learned that some patients with metastasis to the lymph nodes around the common hepatic artery or splenic artery can be cured. Eventually, they saw the dissection of these nodes worthwhile and felt uncomfortable when they did not perform formal D2 dissection on their patients. Since this is how the D2 dissection developed, it is not evidence-based in the sense that it has not been compared with more limited surgery in prospective randomized trials. In that sense, Japanese surgeons should be thankful to their colleagues in Taiwan and the Netherlands who proved or suggested that what they thought was right.

In addition to the wider extent of lymphadenectomy per se, 'classic' D2 dissection contains several additional components such as splenectomy (applicable only for cancer located in the upper third of the stomach) to facilitate dissection of hilar lymph nodes (station Nos. 10 and 11d) and bursectomy to facilitate anatomical dissection of infrapyloric nodes (station No. 6) and to remove microscopic deposits which could develop into peritoneal metastasis. These procedures are time-consuming and add difficulty to an already complex surgical procedure, which leads to increased morbidity. As de Steur et al. [this vol., pp. 96-103] rightly point out, several retrospective studies and small-scale trials as well as subset analyses of the D1 versus D2 trials performed in the Netherlands and Britain have indicated that splenectomy does not improve survival, but it does raise morbidity and mortality. It is the responsibility of the Japanese surgeons who originally proposed splenectomy as a part of D2 dissection to conclude this debate. Final survival analysis of a pivotal randomized trial, JCOG 0110, to confirm the noninferiority of a spleen-conserving strategy will soon take place [7], and the results are eagerly awaited. It is noteworthy, though, that the two occasions of preplanned interim analysis did not result in early termination of the trial. Several surgeons are even more skeptical about the effect of bursectomy to confront peritoneal dissemination. Again, however, the Japanese investigators were reluctant to discard a 'classic' technique without hard evidence against it, especially after looking at an unexpected trend for survival benefit in the bursectomy group in a small- scale randomized trial conducted in Osaka [8]. A largescale phase III trial, JCOG 1001, to clarify this issue was launched and, fortunately, the patient accrual has been beyond expectation.

Regarding PAND, its prognostic benefit was denied by the well-known and acclaimed JCOG 9501 trial only under the condition that (1) nodal metastases to this region were not suspected by preoperative imaging studies, and (2) the patients were treated with surgery alone [9]. It is well-known through numerous retrospective studies that metastases to the para-aortic region are found in 10-20\% of patients with locally advanced gastric cancer who are treated with prophylactic PAND, and that a similar percentage of patients with pathologically confirmed metastases to the para-aortic region survive for more than 5 years after PAND. Thus, it is too early to discard the role of PAND completely from all cases of gastric cancer. If resectable lymphadenopathy is detected in the para-aortic region by preoperative imaging studies, there is currently no answer to the issue of whether the patient should be treated by chemotherapy alone as a systemic disease or by a multimodal strategy that includes surgery with PAND. In Japan, a series of phase II trials testing neoadjuvant chemotherapy with CPT-11/cisplatin [10] and S-1/cisplatin have been conducted for this population with promising outcomes. A new phase II trial is currently ongoing to test a triplet regimen (S-1/docetaxel/cisplatin), and this could eventually develop into a phase III trial with S-1/cisplatin as a tentative standard [Fujitani, this vol., pp. 119-129]. In these trials, the patients are to receive PAND rather than $\mathrm{D} 2$ dissection after the neoadjuvant chemotherapy.

Finally, it is important to come back to the issue of varying incidence of early-stage cancer among different countries when discussing the laparoscopic approach. Gastric cancer surgery with a laparoscopic approach has been challenged throughout the world, and several review articles and meta-analyses of the published studies point to smaller blood loss and increased operating time for the laparoscopic approach along with noninferiority in terms of morbidity and lymph node retrieval [Lee and Yang, this vol., pp. 132-141]. These reports do not immediately bring us to the conclusion that the laparoscopic approach can be considered a standard, however, since it is important to consider the publication bias. There could have been several unsuccessful series that did not reach publication, especially in the West where the disease, especially the resectable types, are uncommon. In contrast, surgeons in the Far East have had a much greater chance to train themselves, owing to the abundance of early-stage 
cancer. The Japanese guidelines recommend systemic nodal dissection even for T1-stage cancer where nodal metastasis is infrequent, and the safety margin of surgery for this population is such that even immature manipulations do not frequently result in treatment failure. There were opportunities for surgeons in the Far East to more comfortably develop both the surgical skills and instruments while keeping untoward consequences to a minimum. Nevertheless, early concerns about port-site recurrences when applying the laparoscopic approach to oncological surgery were greater for gastric cancer than for other malignancies because of the disposition of this cancer type to metastasize through peritoneal dissemination. Adequate clinical trials to explore the long-term outcome of the laparoscopic approach therefore remain mandatory. It should be noted that the randomized trials were conducted in a discreet step-by-step fashion both in Japan and in Korea. In both countries, only early-stage cancers were eligible in the first randomized trial, and more advanced cancers were explored in the second round [11]. It is obviously not practical for Western surgeons to follow the same steps. It may be a good strategy for them to explore safety issues in phase II trials and await the results of the Asian trials.

Eventually, the time might come when laparoscopic surgery should not be supported solely for the self-evident benefit in the short-term and cosmetic outcomes. Clear and magnified views through modern imaging devices have led to more meticulous observation and understanding of the anatomy. Some of the knowledge in surgical anatomy that is currently being unearthed and ac- knowledged in laparoscopic surgery had already been found by the old masters but had long been neglected during the era of open surgery. The laparoscopic approach may now have potential for more meticulous surgery and could be more than 'noninferior' to the open approach if the theoretical benefit in terms of postoperative immunological status turns out to be true. It is currently unknown, though, if surgery at that level can be standardized in Japan where gastric cancer is apt to be treated by general surgeons at small- or intermediate-volume hospitals. Korea or some Western countries may have advantages in this aspect since centralization of gastric cancer treatment have either been accomplished or seriously considered.

To summarize, the abundance of early cancers in the Far East should be taken into consideration when comparing differences in the general concept of gastric cancer as well as treatment strategy between the East and the West. Understanding and updating some key terms such as $\mathrm{D}$ numbers is mandatory for accurate exchange of information. Patients with unresectable or recurrent gastric cancer suffer from poor prognosis across all countries, and it is not productive to consider that cancers in countries with better survival data are simply 'different' from those in other countries. The resources to develop treatment will inevitably depend on the incidence of the disease in each country. It is hoped that the researchers in the Western hemisphere could have a deeper understanding of the situation in the Far East and vice versa for everyone's mutual benefit, and it is hoped that this special issue will encourage such interaction.

\section{References}

1 Aiko T, Sasako M: The new Japanese Classification of Gastric Carcinoma: points to be revised. Gastric Cancer 1998;1:25-30.

2 Sano T, Aiko T: New Japanese classifications and treatment guidelines for gastric cancer: revision concepts and major revised points. Gastric Cancer 2011;14:97-100.

- $3 \mathrm{Wu}$ CW, Hsiung CA, Lo SS, Hsieh MC, Chen JH, Li AF, Lui WY, Whang-Peng J: Nodal dissection for patients with gastric cancer: a randomized controlled trial. Lancet Oncol 2006; 7:309-315.

-4 Siewert JR, Boettcher K, Stein HJ, Roder HD: Relevant prognostic factors in gastric cancer: ten-year results of the German Gastric Cancer Study. Ann Surg 1998;228:449-461.

5 Nakajima T: Gastric cancer treatment guidelines in Japan. Gastric Cancer 2002;5:1-5.
-6 McNeer G, Sunderland DA, McInnes G, Vandenberg HJ Jr, Laurence W Jr: A more thorough operation for gastric cancer: anatomical basis and description of technique. Cancer 1951;4:957-967.

-7 Sano T, Yamamoto S, Sasako M, Japan Clinical Oncology Group: Randomized controlled trial to evaluate splenectomy in total gastrectomy for proximal gastric carcinoma: Japan Clinical Oncology Group study JCOG 0110MF. Jpn J Clin Oncol 2002;32:363-364.

$\checkmark 8$ Fujita J, Kurokawa Y, Sugimoto T, Miyashiro I, Iijima S, Kimura Y, Takiguchi S, Fujiwara Y, Mori M, Doki Y: Survival benefit of bursectomy in patients with resectable gastric cancer: interim analysis results of a randomized controlled trial. Gastric Cancer 2012;15:4248.
-9 Sasako M, Sano T, Yamamoto S, Kurokawa Y, Nashimoto A, Kurita A, Hiratsuka M, Tsujinaka T, Kinoshita T, Arai K, Yamamura Y, Okajima K, Japan Clinical Oncology Group: D2 lymphadenectomy alone or with paraaortic nodal dissection for gastric cancer. N Engl J Med 2008;359:453-462.

-10 Yoshikawa T, Sasako M, Yamamto S, Sano T, Imamura $\mathrm{H}$, Fujitani $\mathrm{K}$, Oshita $\mathrm{H}$, Ito $\mathrm{S}, \mathrm{Ka}$ washima Y, Fukushima N: Phase II study of neoadjuvant chemotherapy and extended surgery for locally advanced gastric cancer. $\mathrm{Br}$ J Surg 2009;96:1015-1022.

-11 Kodera Y, Fujiwara M, Ohashi N, Nakayama G, Koike M, Morita S, Nakao A: Laparoscopic surgery for gastric cancer: a collective review with meta-analysis of randomized trials. J Am Coll Surg 2010;211:677-686. 